\title{
Relapse of Graves' Disease Thirty-Two Years After Treatment with Radioactive Iodine
}

\author{
Khawla F. Ali MD, Keren Zhou MD and M. Cecilia Lansang MD, MPH* \\ Department of Endocrinology, Diabetes and Metabolism, Cleveland Clinic Foundation, 9500 Euclid Avenue / F-20, Cleveland, Ohio, USA
}

*Correspondence to: M. Cecilia Lansang, Department of Endocrinology, Diabetes and Metabolism, Cleveland Clinic Foundation, 9500 Euclid Avenue / F-20, Cleveland, Ohio, Tel: (+1) 216-445-5246; Fax: (+1) 216-445-1656; E-mail: lansanm@ccf.org

Received: September 12, 2017; Accepted: September 20, 2017; Published: September 26, 2017;

\begin{abstract}
Background: Relapse of Graves' disease (GD) after a prolonged period of radioactive iodine (RAI)-induced hypothyroidism is very unusual. We report a case of GD with the longest time-to-relapse so far published, i.e. 32 years after therapy with RAI.

Case Presentation: A 69-year-old woman was referred in 2016 for evaluation of hyperthyroidism. Her history was significant for GD diagnosed at the age of 37. A RAI uptake and scan in 1984 showed an increase uptake of $72 \%$ at 24 hours in a diffusely enlarged gland with no focal lesions. She was treated with $9 \mathrm{mCi}$ of I-131and was rendered hypothyroid requiring levothyroxine therapy. Her hyperthyroidism work-up in 2016 confirmed the first known relapse of her GD. Her thyroid-stimulating hormone level was suppressed at $0.005 \mathrm{uU} / \mathrm{mL}(0.400-5.500 \mathrm{uU} / \mathrm{mL})$, free thyroxine level was elevated at $2.9 \mathrm{ng} / \mathrm{dL}(0.7-1.8 \mathrm{ng} / \mathrm{dL})$, and a RAI uptake and scan showed an increase uptake of $55 \%$ at 22 hours. She was retreated with $10.44 \mathrm{mCi}$ of I-131 therapy, and was rendered hypothyroid again.
\end{abstract}

Conclusion: We report the longest time-to-relapse of GD post-hypothyroidism induced by RAI so far published, i.e. 32 years. Further research is needed to discern the pathophysiology underlying this relapse.

Key words: Graves' disease; Hyperthyroidism; Relapse; Radioactive iodine; Thyroid regeneration

\section{Introduction}

Graves' disease (GD) is the most common cause of hyperthyroidism with an annual incidence of up to 50 cases per 100,000 persons. Radioactive iodine (RAI) therapy has been used in GD for several decades [1]. Persistence or recurrence of GD is occasionally encountered in the immediate post-RAI therapy phase and is likely due to incomplete thyroid tissue ablation. Relapse of GD after a prolonged period of RAI-induced hypothyroidism or achievement of long-term euthyroidism, however, is very unusual. Here, we report the longest case so far published of relapsed GD 32 years post-RAI treatment with a hypothyroid phase in the interim necessitating levothyroxine therapy.

\section{Case Presentation}

A 69-year-old woman was referred in June 2016 for evaluation of hyperthyroidism. She presented with complaints of weight loss, heat intolerance, hair loss and insomnia for several months. Her past medical history was significant for GD diagnosed in 1984. Back then, she had presented with a few months' history of heat intolerance, diarrhea and palpitations. Her exam was notable for a diffusely enlarged thyroid gland with an estimated weight of more than 30 grams. Her work-up in 1984 was remarkable for elevated free thyroxine index (FTI) at $17.7 \mathrm{ug} / \mathrm{dL}$ (reference range: 6.0 - 11.0 $\mathrm{ug} / \mathrm{dL}$ ). A radioactive iodine uptake and scan showed an uptake of $72 \%$ at 24 hours and the gland was diffusely enlarged with no focal lesions consistent with GD. She was treated with $9 \mathrm{mCi}$ of I-131 and was rendered hypothyroid requiring over 10 years of levothyroxine therapy. Her thyroid-stimulating hormone (TSH) was documented at levels as high as $10.05 \mathrm{uU} / \mathrm{mL}$ (reference range: $0.400-5.500 \mathrm{uU} / \mathrm{mL}$ ) in the setting of intermittent non-adherence to levothyroxine therapy between 1985 and 2016 .

Her family history was significant for hyperthyroidism in mother, daughter and maternal aunt. She denied any history of tobacco use. Physical examination upon evaluation in June 2016 showed normal vital signs with a BMI of $34.77 \mathrm{~kg} / \mathrm{m}^{2}$. She had mild right eye proptosis that was not previously noted. Her thyroid gland was slightly enlarged, with an estimated weight of 25 grams.

Her lab work in June 2016 was significant for suppressed TSH of $0.005 \mathrm{uU} / \mathrm{mL}$ and elevated free thyroxine (FT4) of $2.9 \mathrm{ng} / \mathrm{dL}$ (reference range: $0.7-1.8 \mathrm{ng} / \mathrm{dL}$ ). She had been off her thyroid hormone therapy for several months at this point. A thyroid ultrasound demonstrated a heterogeneous, slightly enlarged gland with an inferior right lobar cystic nodule, measuring $9 \times 9 \times 6 \mathrm{~mm}$ ( $\mathrm{L} \times \mathrm{W} \times \mathrm{AP}$ diameter). A thyroid uptake and scan showed homogenous increased uptake of $55 \%$ at 22 hours (normal $10-30 \%$ at 24 -hours) consistent with GD. The patient received a second dose of $10.44 \mathrm{mCi}$ of I-131 therapy in 2016. Subsequent blood work 2 weeks after I-131 therapy demonstrated a decline in FT4 levels from a pre-treatment level of $2.9 \mathrm{ng} / \mathrm{dL}$ to a posttreatment level of $1.8 \mathrm{ng} / \mathrm{dL}$. Thyroid function testing two months 
after RAI treatment revealed an elevated TSH of $16.380 \mathrm{uU} / \mathrm{mL}$, and a low FT4 of $0.2 \mathrm{ng} / \mathrm{dL}$. The patient was restarted on levothyroxine therapy.

\section{Discussion}

Relapse of Graves' hyperthyroidism after RAI-induced hypothyroidism is unusual. Here we report a case of GD with the longest time interval-to-relapse so far published, i.e. 32 years after therapy with I-131, with a hypothyroid phase in the interim that necessitated levothyroxine therapy. In this case, thyroid-stimulating immunoglobulins (TSI) were not obtained as the radioactive iodine uptake and scans were consistent with GD meeting 2016 American Thyroid Association (ATA) guidelines for diagnosis of GD [2,3]. Previously, the longest reported cases of relapsed GD post-RAI induced hypothyroidism were published by Hegele et al. and Tan et al. in 1985 and 1995 respectively. The former presented a case with persistently high levels of TSI who had a relapse of his GD after 23 years of 1-thyroxine therapy post-I-131 hypothyroidism [4]. The latter reported a recurrence of GD in a 90-year-old woman after 22 years of levothyroxine therapy post a $9 \mathrm{mCi}$ ablative dose of I-131 [5]. While relapses of hyperthyroidism shortly after RAI therapy is likely due to incomplete thyroid ablation, this mechanism would be unlikely to explain the relapse of disease several decades later. In this report, we explore a few novel mechanisms underlying the pathogenesis of these rare long-term relapses.

The mechanisms underlying long-term relapses of GD after thyroid ablation have not been fully elucidated, primarily due to scarcity of such cases. A few animal models that simulated the process of thyroid regeneration after its destruction has shed some light on possible pathogenesis of relapses of GD. In the first animal model, experimental mice underwent semi-total partial thyroidectomies (one lobe and 2/5 of the other lobe were resected). In response to a marked decrease in thyroid function, there was an increase in the number of clear, immature cytoplasmic cells expressing 5-bromo2'-deoxyuridine (BrdU), a synthetic nucleoside incorporated into dividing cells and marker of active cell proliferation, in the residual thyroid tissue. Investigators hypothesized that, in response to the stress of surgery, the residual follicular thyrocytes transform into less differentiated clear cytoplasmic cells and subsequently proliferate and differentiate into mature, functional thyrocytes [6]. Whether a similar mechanism exists for tissue regeneration post-RAI ablation is yet unknown.

In another thyroid regeneration mouse model, Experimental Autoimmune Thyroiditis (EAT) mice underwent complete destruction of their follicular architecture by injection of thyroglobulin (Tg) and subsequent induction of endogenous $\mathrm{Tg}$ antibodies. A stem cell marker, Oct-4 mRNA, was detected at baseline suggesting the presence of thyroid stem cells among the mature thyrocytes. After an acute destructive period induced by $\mathrm{Tg}$ antibodies, the murine thyroid gland demonstrated a remarkable capacity for tissue regeneration. There was an increase in BrdU expression suggesting active cell proliferation and concomitant decrease in Oct-4 as the follicles regenerated, elucidating an essential role of pre-existing thyroid stem cells in the regeneration of the thyroid [7].
Although both models emphasize the roles of dedifferentiated thyrocytes and pre-existing stem cells in the regeneration of the thyroid, there may also be a role for differentiated, remnant thyrocytes in the relapse of GD. Remnant thyrocytes that remained viable, but at concentrations insufficient to present clinically with euthyroidism or hyperthyroidism, may have hypertrophied under the influence of chronically elevated TSH levels, in the setting of intermittent noncompliance to levothyroxine therapy. It is well known that in patients with chronic autoimmune thyroiditis, increase in circulating TSH plays a significant role in the development of their goiter. Suppression of TSH by administration of thyroid hormone, on the other hand, has been shown to decrease goiter size [8]. Therefore, it is plausible that the elevation in TSH levels overtime may have stimulated remnant follicular cells, resulting in gland hypertrophy and overproduction of endogenous thyroid hormones.

It is also possible that an elevated TSI level may have also contributed to the hypertrophy of gland overtime. Additionally, a change in the conformational specificity of TSI overtime, resulting in higher affinity to the thyroid-stimulating hormone receptor (TSHR), may also play an important role in the overgrowth of residual differentiated thyrocytes and subsequent relapse of GD [9]. Finally, an acquired gain-of-function mutation within TSHR gene or adenylate cyclase-stimulating $\mathrm{G}$ a protein gene, a downstream signal in the TSHR pathway, could also explain relapse of GD. Although plausible, such mutations would more likely result in patchy, nodular overgrowth, similar to what has been described in development of toxic thyroid nodules, rather than a diffusely-enlarged gland [10]. Whether these mutations could develop as long-term sequelae of radioiodine exposure is unknown.

\section{Conclusions}

We reported a case of GD that relapsed 32 years after initial treatment with RAI-therapy with a documented hypothyroid phase in the interim that necessitated levothyroxine therapy. The mechanism behind this relapse is not fully understood. Further basic science and clinical research is needed to discern the pathophysiology underlying this rare relapse.

\section{Acknowledgements}

An abstract on this case was accepted for a poster presentation at the national meeting of The Endocrine Society; April 3rd, 2017; Orlando, FL, USA.

Disclosure Statement: The authors declare that there is no conflict of interest regarding the publication of this paper.

\section{References}

1. Smith TJ, Hegedus L. Graves' disease. N Engl J Med 375: 1552-1565. [Crossref]

2. Wartofsky L, Glinoer D, Solomon B, Nagataki S, Lagasse R, et al. (1991) Differences and similarities in the diagnosis and treatment of Graves' disease in Europe, Japan, and the United States. Thyroid. 1(2): 129-35. [Crossref]

3. Ross DS, Burch HB, Cooper DS, Greenlee MC, Laurberg P, et al. (2016) 2016 American Thyroid Association Guidelines for Diagnosis and Management of Hyperthyroidism and Other Causes of Thyrotoxicosis. Thyroid 26(10): 1343-1421. [Crossref]

4. Hegele RA, Volpé R (1985) Relapse of Graves' disease 23 years after treatment with radioactive iodine (131I). J Clin Lab Immunol 18(2): 103-105. [Crossref] 
5. Tan GH, Gharib H (1995) Recurrent hyperthyroidism after radioiodine-induced hypothyroidism: report of two cases and literature review. Endocr Pract 1(3): 158-160. [Crossref]

6. Kimura S (2014) Thyroid Regeneration: How Stem Cells Play a Role? Frontiers in Endocrinology 5: 55.

7. Chen CY, Kimura H, Landek-Salgado MA, Hagedorn J, Kimura M, et al. (2009) Regenerative potentials of the murine thyroid in experimental autoimmune thyroiditis: role of CD24. Endocrinology 150(1): 492-429. [Crossref]

8. Berghout A, Wiersinga WM, Drexhage HA, Smits NJ, Touber JL (1990) Comparison of placebo with L-thyroxine alone or with carbimazole for treatment of sporadic non-toxic goitre. Lancet 28: 336(8709): 193-7. [Crossref]

9. McLachlan SM, Rapoport B (2013) Thyrotropin-blocking autoantibodies and thyroid-stimulating autoantibodies: potential mechanisms involved in the pendulum swinging from hypothyroidism to hyperthyroidism or vice versa. Thyroid 23(1): 14-24. [Crossref]

10. Liu C, Wu C, Wang F (2010) Mutations of GNAS and TSHR genes in subclinical toxic multinodular goiter. Ann Otol Rhinol Laryngol 119(2): 118-124.

\section{Abbreviations}

GD: Graves’ Disease

RAI: Radioactive iodine

FTI: Free Thyroxine Index

TSH: Thyroid-Stimulating Hormone

FT4: Free Thyroxine

TSI: Thyroid-Stimulating Immunoglobulin

EAT: Experimental Autoimmune Thyroiditis

Tg: Thyroglobulin

TSHR: Thyroid- Stimulating Hormone Receptor

\section{Citation:}

Khawla F. Ali, Keren Zhou and M. Cecilia Lansang (2017) Relapse of Graves' Disease Thirty-Two Years After Treatment with Radioactive Iodine. Endocrinol Diabetes Metab $J$ Volume 1(3): 1-3 